\title{
PROCESSO GRUPAL EM ENFERMAGEM: POSSIBILIDADES E LIMITES 1
}

\author{
GROUP PROCESS IN NURSING: POSSIBILITIES AND LIMITS.
}

Denize Bouttelet Munari ${ }^{2}$

RESUMO: Este trabalho descreve nossa trajetória de estudo e reflexão sobre a utilização de grupos na assistência. Através de uma perspectiva qualitativa buscamos identificar, na percepção dos entrevistados, aspectos que os motivam para essa atividade, suas fontes de aprendizagem e os pontos relevantes da sua experiência concreta com grupos. Verificamos que os enfermeiros reconhecem o valor terapêutico desse trabalho provendo-lhe condições físicas e estruturais adequadas. No entanto, observamos que um dos fatores limitantes no desenvolvimento dessa atividade é a dificuldade no manejo de situações grupais que revelam os meandros do sentimento humano, indicando que o enfermeiro coordenador de grupo necessita, além de recursos teóricos, exercitar o seu autoconhecimento para prover um ambiente e relacionamento grupal, capazes de otimizar o valor terapêutico dessa atividade.

UNITERMOS: Grupos - Enfermagem - Atividade grupal.

\begin{abstract}
This paper describes our study trajectory and reflection on the use of groups in Care. Through a qualitative perspective we aim at identifying, under the interviewees perception, the aspects which motivate them for this activity, their learning sources and their group concrete experiences relevant topics. We have verified that nurses recognize the therapeutic value of this work which promotes them adequate structural and physical conditions. However, it has been observed that one of the limiting factor for this activity is the difficulty of dealing with group situations which reveal the nuances of human feeling indicating that the group coordinator nurse besides needing theoretical resources, he also needs to exercise his self-knowledge in order to provide a group relationship and environment able to optimize this activity therapeutic value.
\end{abstract}

KEYWORDS: Groups - Nursing - Group activity.

\footnotetext{
${ }^{1}$ Resumo de tese apresentado à Escola de Enfermagem de Ribeirão Preto-Universidade de São Paulo para a obtenção do grau de Doutora, sob orientação da $\operatorname{Prof}^{a} \mathrm{Dr}^{\mathrm{a}}$ Antônia Regina Furegatto Rodrigues.

2 Professora Adjunta do Centro de Graduação em Enfermagem da Faculdade de Medicina do Triângulo Mineiro Uberaba-MG.
} 


\section{INTRODUÇÃO}

Grande parte das atividades desenvolvidas pelos seres humanos é realizada em grupos. Assim sendo, estudar os grupos é de uma importância incontestável. Os pesquisadores sobre 0 assunto, em geral, iniciam suas exposições destacando a natureza gregária do homem, assim como as relações entre os individuos e grupos (Cartwright \& Zander, 1967; Mailhiot, 1981; Osório e col., 1986). Percebemos, portanto, quão antiga é essa característica do ser humano, anterior mesmo à sua consciência da necessidade de viver como um ser agregado. Sendo assim, o homem sempre utilizou o grupo para trabalhar, divertir, estudar.

Durante a nossa trajetória de aproximação frente à temática, focalizamos nosso interesse na utilização do grupo como um recurso para ajudar e assistir pessoas.

A literatura relata o trabalho desenvolvido por Pratt no Hospital Geral de Massachussets, nos EUA, em 1905, como marco histórico no que concerne às primeiras experiências de utilização de grupos enquanto recurso terapêutico, apontado na seguinte citação:

" depois da introdução do método de grupo por Pratt e seus seguidores para enfrentar a influência de emoções em doenças somáticas, o emprego dessa técnica começou a difundir-se amplamente" (KADIS e col.,1967: 21)

Segundo Cappon (1978), as investigações de Pratt foram desenvolvidas, inicialmente, com pacientes tuberculosos através de reuniões de pacientes em grupos para informá-los sobre sua doença e as medidas higiênicas necessárias para sua recuperação. Mais tarde, o pesquisador utilizou o mesmo método para pacientes diabéticos e cardíacos. O fundamento básico desse método partia do pressuposto de que os resultados do tratamento dependia da influência benéfica de uma pessoa sobre a outra. Wood (1990) refere-se a esse trabalho como um espaço onde a solidão, o pessimismo, a depressão e outros efeitos psicológicos advindos da doença poderiam ser aliviados pelo suporte emocional existente em um grupo estável de pessoas com preocupações e experiências semelhantes.

$\mathrm{Na}$ área de enfermagem, a utilização de grupos não se constitui propriamente em uma novidade. Por natureza, o enfermeiro é um profissional que desenvolve o seu trabalho em grupo como, por exemplo, na equipe de enfermagem, durante a passagem de plantão, executando atividades educativas, e no ensino, realizando grupos de discussão de casos ou como estratégia em disciplinas em que o grupo é visto como parte da aprendizagem. 
Verificamos na literatura nacional que o atendimento de enfermagem em grupo tem sido utilizado em várias áreas, sendo a enfermagem de saúde pública, na década de 70 a pioneira na publicação dos resultados de suas investigações partindo da utilização dessa modalidade na assistência a gestantes. (Duarte \& Muxfeldt, 1975 e Schimidt \& Freddi, 1975).

Porém, é a partir da década de 80 que pudemos encontrar várias pesquisas registradas em periódicos nacionais relatando os trabalhos realizados por enfermeiros através de atividades grupais atendendo a uma variada clientela, tais como pessoas com problemas de locomoção, de hanseniase, de obesidade, de cardiopatias, grupos de mães, de adolescentes, de diabéticos, conforme salientam respectivamente Leite; Aguiar; Carvalho; 1981, Cristofolini, 1982, Santos \& Mendes, 1983, Vargas \& Scain, 1983, Corrêa \& Gebara (1984), Martins \& Moretto (1985) e Scain (1986).

A literatura internacional por sua vez é vastíssima a respeito da temática, cujo maior número de trabalhos é encontrada na enfermagem norte-americana, que se encontra respaldada no " berço de nascimento" dos estudos de grupo liderados por Kurt Lewin na década de 30 (Mailhiot 1981. Destacamos aqui os trabalhos de Loomis (1979), Wilson 1985, Heyney \& Wells (1989), Alley \& Foster (1990), Lewis; Frain \& Donnelly (1993) e Payne (1993).

Porém um aspecto chamou-nos a atenção, quando notamos que os trabalhos publicados pela enfermagem brasileira na sua grande maioria não se referem à questão do preparo do enfermeiro para o desenvolvimento dessa tarefa como requisito para a sua realização.

Sabemos, por nossas experiências com alunos de graduação, pósgraduação em Enfermagem e por nossa própria vivência, que essa não se constitui tarefa das mais fáceis, embora agradável e muito compensadora.

Pesquisas anteriores realizadas por nós demonstraram que os profissionais têm disponibilidades diferentes ao entrarem para o trabalho grupal. Esse fato interfere no seu aproveitamento do processo, e conseqüentemente pode trazer ao participante experiências satisfatórias ou não (Campos, Munari, Loureiro \& Japur, 1992; Loureiro; Japur, Campos \& Munari, 1990 e Japur, Loureiro, Campos \& Munari, 1992).

Por essa razão, acreditamos que precisamos conhecer quais são os aspectos importantes na formação do enfermeiro para que o mesmo possa realizar atividades em grupo de forma mais consciente, reflexiva e efetiva. Em uma pesquisa preliminar, cujos resultados apresentamos na $46^{\circ}$ Reunião Anual da SBPC, observamos que do ponto de vista formal (através de cursos, especialização, supervisão e outros) os enfermeiros tiveram muito pouca oportunidade de conhecer aspectos teórico-vivenciais sobre o trabalho grupal 
(Munari, 1994). Essa pesquisa permitiu-nos ainda uma aproximação com a clientela entrevistada de tal forma que passamos a levantar novos questionamentos.

Tais questões emergiram do nosso interesse em conhecer mais detalhadamente os elementos que o enfermeiro utiliza na condução de atividades grupais, e de que forma adquiriu ou adquire habilidades para isso. Portanto, partindo da percepção que o enfermeiro tem sobre sua atuação como coordenador de grupos na assistência, os objetivos desta investigação foram: 1 Identificar os enfermeiros que desenvolvem atividade grupal na assistência, cuja perspectiva do seu trabalho vislumbra o grupo como um recurso terapêutico; 2 Apreender os aspectos que motivam o enfermeiro a assistir pessoas através de grupos; 3 - Conhecer as fontes de aprendizagem que o enfermeiro utiliza para desenvolver essa atividade; 4 - Identificar os pontos relevantes no desenvolvimento dessa tarefa, a partir da experiência concreta do enfermeiro.

\section{METODOLOGIA}

O interesse sobre essa temática e sua relação com o trabalho do enfermeiro levou-nos, num primeiro momento, a buscar, na prática, informações que nos permitissem observar a inserção das atividades grupais no cotidiano do enfermeiro. Para tanto, realizamos um levantamento para conhecermos a dimensão deste trabalho do enfermeiro no município de Ribeirão Preto.

O objetivo desse levantamento foi subsidiar a realização de entrevistas, caracterizando estas atividades em profundidade e sob a perspectiva do enfermeiro enquanto agente. O levantamento foi tratado como um estudo exploratório, uma vez que este prestou-se a oferecer ao pesquisador elementos para aprofundar o seu conhecimento acerca de um fenômeno, e como uma etapa de um processo de pesquisa que prevê desdobramentos (Selltiz e cols., 1975).

Os dados obtidos pelo levantamento permitiu-nos identificar três tipos de trabalho desenvolvidos pelos enfermeiros. O primeiro deles denominamos grupos de caráter informativo e de educação em saúde; o segundo, grupos para a manutenção de programas compulsórios; e finalmente, o terceiro, grupos de reabilitação e apoio emocional.

Em função dos objetivos estabelecidos para essa pesquisa, optamos por restringir a amostra aos enfermeiros envolvidos com o terceiro grupo, cujas características descrevemos a seguir. 


\section{GRUPOS DE REABILITAÇÃO E APOIO EMOCIONAL}

Objetivo - tem como linha mestra o trabalho de reabilitação que implica no físico, social e psicológico, podendo contemplar todas as instâncias ou algumas delas com ênfase no potencial do grupo para ajuda entre as pessoas.

Periodicidade dos encontros - no geral, são encontros semanais ou mensais, porém com funcionamento permanente permitindo ao participante estar em contato enquanto necessita.

Tipo de participante - é caracterizado por uma clientela fixa na maioria das vezes, constituindo-se em grupos fechados, permitindo no entanto que o participante, que não está em contato com o serviço, retorne quando tem necessidade.

Tipo de coordenação - o coordenador é fixo ou se reveza entre pessoas que são conhecidas pelos participantes e que acompanham o trabalho.

Definição da temática - é livre para que os participantes definam o assunto que querem tratar no dia.

\section{ATIVIDADES INCLUÍDAS NESSA CATEGORIA}

- GRUPOS DE MULHERES MASTECTOMIZADAS (02)

- GRUPO DE FAMILIARES DE PESSOAS AIDÉTICAS (01)

- GRUPO DE PESSOAS EPILÉPTICAS (01)

- GRUPO DE PACIENTES PSIQUIÁTRICOS CRÓNICOS (01)

- GRUPO DE PESSOAS LARINGECTOMIZADAS (01)

- GRUPO DE PESSOAS COLO, ÍLEO OU UROSTOMIZADAS (01)

- GRUPO DE PESSOAS OBESAS (01)

- GRUPO DE PESSOAS IDOSAS (01)

- TOTAL DE TRABALHOS $=(09)$

Os aspectos metodológicos que envolviam a condução dessa investigação estavam de acordo com as diretrizes preconizadas por Bogdan \& Biklen ( 1982). Atentas aos recursos metodológicos que pudessem permitir-nos uma leitura qualitativa dos dados, observamos que

"numa busca qualitativa, preocupamo-nos menos com a generalização $e$ mais com o aprofundamento e abrangência da compreensão seja de um grupo social, de uma organização (...) " (Minayo, 1993: 102) 
A nossa tentativa em conhecer a perspectiva dos enfermeiros acerca da coordenação dos grupos levou-nos a uma segunda etapa de aproximação dos sujeitos. Nesta fase utilizamos uma entrevista com questões abertas (Anexo I), cujo roteiro continha tópicos que serviram de guia ou apoio na condução das mesmas.

A primeira análise das entrevistas fazíamos logo após a coleta, ouvindo-as e transcrevendo em seguida. Neste momento podiamos perceber a ênfase dada pelo entrevistado a cada pergunta, e tentamos identificar possiveis pistas para o estabelecimento de categorias. Bogdan \& Biklen (1982) denominam de" categorias de código" as palavras ou frases que aparecem com certa regularidade ou padrão.

Após a transcrição de todo o material, passamos a utilizar o software "The Ethnograph", idealizado pelo Dr. John Seidel (Universidade de Colorado - USA) e Dr. Jack Clark (Universidade de Harvard), que proporcionou rapidez e eficiência no manejo dos dados. Esse programa tem sido utilizado por pesquisadores da área da enfermagem como um valioso recurso para se trabalhar com investigações que necessitem desse tipo de manejo de dados, apontado por Taft (1993) como um substituto dos procedimentos mecânicos como a utilização de xerox, recortes, cola e tesoura. Cassiani (1993), Zago (1994) e Medeiros (1995) discorrem sobre o seu uso no processo de organização de dados para a análise, suas vantagens e formas de proceder.

Para o desenvolvimento dessas categorias, empregamos os princípios sugeridos por Bogdan \& Biklen (1982), os quais conduzem à análise e ao desenvolvimento de categorias codificadoras. Os autores apresentam várias possibilidades de se codificar o material coletado, que podem servir de apoio e guia nessa etapa. Alguns são mais apropriados que outros, podendo se lançar mão de mais de um deles ou misturá-los e adequá-los de acordo com a natureza da pesquisa.

\section{ANÁLISE E DISCUSSÃO DOS DADOS}

O caminho metodológico mostrou-se adequado às nossas necessidades, permitindo-nos um olhar cuidadoso para questões que nos inquietavam, mostrando que estudar um recorte da vivência dos enfermeiros na coordenação de grupos para assistir pessoas sobre a perspectiva deles próprios é uma possibilidade para entendermos a sua prática.

Os sujeitos deste estudo foram enfermeiros, vinculados a Instituições Públicas de Saúde nas áreas hospitalar e ambulatorial no município de Ribeirão Preto - SP. 
A imersão nos dados e as tentativas de identificação dos códigos, seguindo as sugestões de Bogdan \& Biklen (1982) para a realização dessa etapa de pesquisa, levou-nos ao reconhecimento de uma categoria central que denominamos de GRUPOS ENQUANTO UMA OPÇÃO NA ASSISTÊNCIA DE ENFERMAGEM.

A leitura dos dados codificados feita sob um constante exercício em relacioná-los com os aspectos teóricos que esboçamos como suporte para esse estudo e somados aos objetivos que estabelecemos para a investigação levounos a vislumbrar o aparecimento de duas categorias mais específicas partindo da categoria central, ou seja, ENFERMEIROS COORDENADORES DE GRUPOS NA ASSISTÊNCIA Y O TRABALHO GRUPAL NA VIVÊNCIA DO ENFERMEIRO.

As categorias foram estudadas e desdobradas em subcategorias por contextualizarem aspectos relevantes para análise. No presente artigo focalizamos aqueles mais importantes.

\section{ENFERMEIROS COORDENADORES DE GRUPOS NA ASSISTÊNCIA}

Com esta categoria buscamos caracterizar o enfermeiro que realiza o grupo do ponto de vista da sua motivação para o trabalho e das fontes de aprendizagem de que dispõe para isso.

O modo como os enfermeiros aproximaram-se do trabalho grupal revelou-nos que alguns atendem às necessidades que emergem nos serviços, os quais trabalham conforme expressam nas seguintes falas:

"Na verdade esse trabalho já existia bem antes de eu entrar no ambulatório, o Farmacêutico me convidou para coordenar com ele porque ele estava sozinho e eu aceitei. " (E - 07)

" O grupo de obesidade já estava formado, estava pronto, eu já o conhecia desde a graduação quando trabalhei com a coordenadora. Quando eu cheguei como funcionária do Centro de Saúde, a pessoa que coordenava saiu e eu assumi o grupo; ele não surgiu da minha iniciativa. " ( $E$ - 08)

Um outro modo é buscando uma abordagem do ser humano pressupondo sua integralidade, mostrando que os enfermeiros, sujeitos desta investigação, estão atentos para adquirirem uma postura em direção à modernidade, ressaltando a qualidade de vida como um importante, senão o primeiro, indicador de saúde. Os trechos a seguir ilustram esse fato:

A minha motivação é estar tentando mostrar para essas mulheres que, por pior que seja a situação delas, o importante é o momento que ela está 
vivendo. (...) para mim está claro, hoje, que não pretendemos a cura, e sim melhorar a qualidade de vida delas..." (E - 02)

"A forma como estamos trabalhando nos leva a acreditar que as pessoas vão ficar menos doentes, mais saudáveis, viver na sua casa, e isso é qualidade de vida" (E - 05)

“ (...) os problemas dos laringectomizados depois que eles saiam do hospital eram muito piores do que quando ele estaza lá. Então nós percehemos que esse era o momento que a gente precisava dar mais apoio para o paciente, pelo menos ouvílo para que ele pudesse ter alguém pra conversar que entendesse e que pudesse ajudar de alguma forma (...)" (E-06)

Nossa experiência corrobora de certa forma esses achados, uma vez que compreendemos a importância da existência desses trabalhos para atender o ser humano numa dimensão total. Do ponto de vista de Mello Filho (1986 : 280)

" hoje, a possibilidade de atender em grupos doentes somáticos portadore's de doenças crônicas configura, ao nosso ver, uma das direções revolucionárias de nossas práticas de saúde e do atendimento em massa de nossas populações. É um trabalho que apenas engatinlıa, diante de enormes possibilidades que se abrem ao seu futuro."

Quanto às fontes de aprendizagem, percebemos que no todo das falas fica explícito que a realização dos grupos é baseada quase que exclusivamente em experiências e vivências, evidenciadas pelos entrevistados como recurso para a execução da atividade.

"A gente tira muita coisa é do próprio paciente, com cada grupo a gente aprende alguma coisa" (E - 04)

"Acho que só na fala das pacientes, das dificuldades delas que eu encontrei durante a condução dos grupos, eu acho que aprendi com o grupo." (E - 08).

As fontes de aprendizagem relacionadas aqui pelos enfermeiros são basicamente oriundas das experiências e vivências com o grupo. Nesse sentido, o profissional parte do empírico buscando acertar e demonstra, no seu discurso, muita tranqüilidade em expor a sua real situação.

Acreditamos que não é possivel negar a contribuição da experiência para a formação do profissional que trabalha com grupos, porém contar apenas com esse recurso para lidar com a emergência de sentimentos e emoções pode trazer dificuldades ao coordenador, que estaria mais resguardado se tivesse algum conhecimento da dinâmica humana e dos grupos. Além disso, teria mais segurança quanto ao planejamento, à condução do grupo, aos problemas 
apresentados pelos elementos que o compõem e quanto à própria avaliação do trabalho executado, podendo utilizar melhor o potencial terapêutico do grupo.

A questão da formação do enfermeiro para o manejo de grupos é criticada pelos próprios entrevistados que identificam os seus limites e revelam a necessidade de embasamento para algumas, na própria graduação. Lopes (1992,: 86) faz considerações semelhantes acerca da formação do enfermeiro para atividade dessa natureza dizendo que " não saímos do curso de graduação, nem da especialização ou ainda da pós-graduação com know how para dirigirmos grupos". Isso nos indica a real importância de buscarmos embasamento para o desenvolvimento da função de coordenador de grupo, que inicia ao nosso ver com a percepção do enfermeiro sobre sí mesmo (autoconhecimento) e sobre o modo como ele se relaciona grupalmente. A fala seguinte relata a percepção de um dos enfermeiros sobre essa questão.

\footnotetext{
"Acho que a gente está aprendendo a modificar nossa postura de poder chorar junto das pacientes porque a gente acaba criando um vínculo que não é só profissional, não é amizade, é complexo (...) É importante o profissional ir buscar essa capacitaçño para trabalhar com grupo porque nós não temos isso na nossa formação e quando a gente começa fazer o grupo é que vê a dificuldade." (E-02)
}

Nossa experiência atual no Curso de Graduação em Enfermagem, ministrando disciplina introdutória sobre a Dinâmica de Grupos, permitiu-nos perceber que a maioria dos alunos se surpreende com sua complexidade e com as possibilidades de utilização desses conhecimentos na profissão. Ressaltam a importância e a necessidade da disciplina ser oferecida nos primeiros semestres do Curso para que possam testar sua aplicação nos estágios.

Acreditamos que o fato de despertarmos o aluno para essas questões e reforçarmos a necessidade do autoconhecimento e de suporte teórico, planejamento adequado e, principalmente, a importância da supervisão venha contribuir para a expansão da utilização de grupos na assistência, ou para outras intervenções de enfermagem. Esperamos, entretanto, estarmos também sensibilizando futuros enfermeiros para uma atuação mais consciente, fundamentada e capaz de aproveitar suas potencialidades.

\section{O TRABALHO GRUPAL NA VIVÊNCIA DO ENFERMEIRO}

Com o intuito de identificar, junto aos enfermeiros coordenadores de grupos na assistência, quais os pontos relevantes no desenvolvimento de grupos partindo da sua experiência concreta, tentamos fazer uma leitura dos dados coletados nas entrevistas que privilegiassem o que Bogdan \& Biklen (1982) chamaram de códigos de estratégias, que se referem às táticas, métodos, caminhos, técnicas que os sujeitos utilizam na realização de suas tarefas. 
Utilizamos também como guia o referencial de Loomis (1979), considerando que o mesmo nos oferece suporte para a compreensão teórica do processo pelo qual o enfermeiro desempenha a função de coordenador de grupo.

Notamos, à medida que os enfermeiros vão descrevendo a realização dos grupos, que existe uma certa semelhança com as etapas do trabalho grupal definidas por Loomis (1979), especialmente no que diz respeito ao planejamento de condições estruturais do trabalho.

Quanto aos objetivos, observamos através das entrevistas que todos os grupos se constituem em espaço para que a clientela, consideradas as suas diferenças, tivesse oportunidade de ventilar suas dificuldades beneficiando-se do que Yalom (1975) ${ }^{35}$ denominou de "fatores curativos" do grupo.

"O grupo é um espaço para que as mulheres coloquem tudo pra fora, a gente về que são pessoas que não têm quem lhes dê atenção, elas entram tímidas e de repente começam a se colocar de maneira até forte demais, colocando suas particularidades, e a gente vêe que isso é necessário e que é importante pra elas." (E-01)

O local e horário dos grupos, assim como 0 número de coordenadores/terapeutas, de clientes que fazem parte do grupo e da extensão dos encontros, são considerados importantes variáveis físicas que fazem parte da estrutura que caracteriza o grupo (Loomis, 1979).

Observamos que, para a maioria dos enfermeiros, estes itens são considerados no manejo dos grupos, demonstrando a responsabilidade do coordenador em prover um espaço adequado, com conforto, compromisso com os horários, características essas que oferecem segurança ao grupo (Loomis, 1979; Heyney \& Wells, $1989^{10}$ ). O exemplo seguinte demonstra a preocupação do enfermeiro com o grupo que coordena:

“ A gente faz o grupo no retorno mensal dos pacientes, o horário procuramos adaptar dentro do interesse deles. Procuramos manter as cadeiras em círculo, aliás todas as atividades que a gente tem feito, temos procurando usar essa disposição das cadeiras para dar abertura para as pessoas falarem (...). Nós procuramos começar as reuniões no horário e nunca ultrapassar uma hora e meia, duas horas no máximo." (E - 04)

A maneira como os enfermeiros demonstram compreender e trabalhar as nuances do grupo indica que problemas comuns no cotidiano dos grupos são vivenciados com dificuldades. Possivelmente isso ocorre por desconhecimento do manejo adequado dessas situações, que seriam bastante simplificadas com um embasamento teórico e supervisão ou com outros recursos do próprio enfermeiro. Nas falas que se seguem notamos esses aspectos: 
"Para mim a maior dificuldade era saber se o que eu fazia estava certo ou errado. Eu me via numas encruzilhadas muito grandes, não sabia como lidar com a questão do reconhecimento." (E - 08)

"Eu acho que a gente poderia ter uma formação melhor com relação ao trabalho em grupo, não sei se é a gente ir buscar ou se dentro da própria escola, dentro da formação do enfermeiro já ter alguma coisa para as pessoas despertaremi." (E-04)

A busca de uma formação adequada que não se encerra, no nosso ponto de vista, com recursos teóricos poderiam tranquilizar os coordenadores e, com isso, os enfermeiros poderiam estar mais seguros da utilização dos "fatores curativos", descritos por Yalon (1975) e possivelmente teriam mais claros para si mesmos os fundamentos do manejo terapêutico do grupo com todas as suas possibilidades.

Fica evidente a luta que precisa ser travada para a manutenção dessas atividades, uma vez que o enfermeiro precisa demonstrar e exercitar sua competência no manejo do grupo e ao mesmo tempo convencer outros enfermeiros e a própria instituição em que trabalha de que essa é uma atividade necessária.

O interesse para que o grupo aconteça é do enfermeiro com a aquiescência relativa da instituição em que ele trabalha, cuja contrapartida na maioria das vezes é insignificante, muitas vezes colocando obstáculos como não provendo espaço físico adequado, material permanente e/ou de consumo ou ainda dificultando a participação do enfermeiro.

O que encontramos na literatura nacional especifica de enfermagem sobre a utilização de grupos na assistência tem, na sua maioria, semelhança com os resultados obtidos nesse trabalho. Grande parte das publicações relatam experiências da utilização desse recurso sem, no entanto, discutir sua fundamentação.

\section{CONSIDERAÇÕES FINAIS}

A concretização deste trabalho possibilitou-nos compreender a importância de pesquisarmos essa temática como forma de oferecer embasamento aos enfermeiros que trabalham com grupos na assistência.

É importante ressaltar que, durante a trajetória da investigação e o nosso envolvimento com os enfermeiros, observamos o compromisso e dedicação com que esses profissionais realizam os grupos junto aos pacientes. 
Pudemos perceber que os enfermeiros reconhecem o valor terapêutico da atividade grupal como um recurso importante procurando, dentro do que lhes é possivel, prover de forma adequada as condições para a sua efetivação.

No entanto, o esforço em realizar o que de melhor eles podem fazer tem esbarrado em seus próprios limites e dificuldades concretas de manejar a situação grupal. Tais obstáculos são provenientes muitas vezes da falta de preparo específico para trabalhar situações dessa natureza, como os próprios entrevistados nos revelaram.

Porém, essa questão não é a causa única das dificuldades, pois, como observamos, o enfermeiro identifica que os recursos teóricos são fundamentais para a condução do grupo, mas que ao longo de suas experiências foi preciso, sobretudo, repensar a sua postura profissional como um todo frente aos problemas relatados pelos pacientes.

A reflexão sobre a formação do enfermeiro é questão também trazida pelos entrevistados, que revelam a necessidade de buscarmos uma postura mais adequada frente ao relacionamento do enfermeiro com os pacientes, colegas e outros profissionais, que se contraponha àquela que determina que o enfermeiro seja um profissional imparcial e onipotente frente à "dor" das pessoas. Esse novo posicionamento deve estar presente no modo como ensinamos os alunos ainda na graduação.

Segundo os enfermeiros, suas experiencias e dificuldades com as pessoas nos grupos mostraram que é preciso e possivel desmistificar essa figura do profissional rígido, cujo relacionamento é extremamente distante, para uma postura de um personagem crítico e flexível, capaz de orientar a trajetória da sua história. 


\section{ANEXO I - ROTEIRO DE ENTREVISTA}

1. O que motivou você para a realização desse trabalho com grupo?

2. Para que você desenvolva essa tarefa, quais foram ou têm sido suas fontes de aprendizagem? Onde busca elementos para o seu aperfeiçoamento?

3. Quando você faz uma reunião, considere o seguinte:
A) O que você considera serem condições favoráveis?
B) O que você considera serem fatores que dificultam?
C) Como você avalia os resultados do grupo?
D) Você sente falta de alguma coisa para realizá-los?

4. Você vê alguma perspectiva para o futuro desse trabalho?

5. Você pensa em realizar alguma mudança? 


\section{REFERÊNCIAS BIBLIOGRÁFICAS}

1. ALLEY, N. M. \& FOSTER, M. C. Using self-helps support groups: a framework for nursing pratice and research. Journal of Advanced Nursing, n. 15, p. $1383-8,1990$.

2. BOGDAN, R. C. \& BIKLEN, S. K. Qualitative research for education. Na introduction to theory and methods. Boston: Allyn and Bacon Inc., 1982.

3. CAMPOS, M. A .; MUNARI, D. B.; LOUREIRO, S. R. \& JAPUR, M. Dinâmica de grupo: reflexões sobre um curso teórico-vivencial. $R$. Tec. Educ., v. 108, n. 21, p. $41-9,1992$.

4. CAPPON, J. El movimento de encuentro en psicoterapia de grupo: descripción y análisis critico. Mexico: Trillas, 1978.

5. CARTWRIGHT, D. \& ZANDER, A . Dinâmica de Grupo: pesquisa e teoria I e II. São Paulo: EPU/EDUSP, 1967.

6. CASSIANI, S. H. B. Buscando significado para o trabalho: o aperfeiçoamento profissional sob a perspectiva de enfermeiras. Ribeirão Preto, Universidade de São Paulo.

7. CORREAA, G. M. \& GEBARA, O . - Higiene dentária - relato de uma experiência com grupo de mães. R. Bras. Enferm. Brasília, v. 37, n. 3 / 4, p. 228-36, 1984.

8. CRISTOFOLINI, L. Prevenção de Incapacidade na Hanseníase. R. Bras. Enferm. Brasília, v. 35, n. 3/4, p. 226-37, 1982.

9. DUARTE, N. M. N. \& MUXFELDT, L. C. O papel da enfermeira na assistência à gestante sadia. R. Bras. Enferm. Brasília, v. 28, n. 4, p. 70-4, 1975.

10. HEINEY, S. P. \& WELLS, L. M. Strategies for organizing and maintaining successful support groups. Oncology Nursing Forum, v. 16, n. 2, p. 803-9, 1989.

11. JAPUR, M.; LOUREIRO, S. R.; CAMPOS, M. A . \& MUNARI, D. B. - A aprendizagem teórico-vivencial: exploração de alguns limites $e$ possibilidades. Paidéia, n. 2, p. 51-60, 1992.

12. KADIS, A . L. e cols. Psicoterapia de grupo. São Paulo: Ibrasa, 1967. 
13. LEITE, J. L.; AGUIAR, R. C. B. \& CARVALHO, M. L. A relação de ajuda a um grupo de clientes com problemas de locomoção. R. Bras. Enferm. Brasilia, v. 34, n. 3 / 4, p. 211-223, 1981.

14. LEWIS, D. J.;FRAIN, K. A . \& DONNELLY, M. H. Chronic Pain Management Support Group: a program designed to facilitate coping. Rehabilitation Nursing, v. 18, n. 5, sept./oct., 1993.

15. LOOMIS, M.E - Group process for nurses. Saint Louis: Mosby Company, 1979.

16. LOPES, M. E. E. F. L. - Grupo de espera com familiares de pacientes psiquiátricos. Ribeirão Preto, 1992. 116 p. Dissertação (mestrado) Escola de Enfermagem de Ribeirão Preto - USP.

17. LOUREIRO, S. R.; JAPUR, M.; CAMPOS, M. A . \& MUNARI, D. B. - A produção gráfica coletiva de um grupo de alunos como elemento projetivo de expectativas e da avaliação do processo grupal. In: III Encontro de pesquisadores em saúde mental e II Encontro de enfermeiros psiquiátricos. Anais... Ribeirão Preto: 1990. P. 124-141.

18. MAILHIOT, G. B. Dinâmica e gênese dos grupos. São Paulo: Duas Cidades, 1981.

19. MARTINS, Y. P. \& MORETTO, S. C. Educação para a saúde junto a um grupo de adolescentes: relato de uma experiência de estudantes de enfermagem. R. Bras. Enferm. Brasília, v. 38, n. 3/4, p. 370-9, 1985.

20. MEDEIROS, M. "Percepções dos atores sociais que coordenam programas assistenciais às crianças e aos adolescentes em situação de rua no município de Ribeirão Preto, SP. Ribeirão Preto, 1995. 86 p. Dissertação (Mestrado) - Escola de Enfermagem de Ribeirão Preto, Universidade de São Paulo, 1995.

21. MELLO FILHO, J. Grupoterapia com pacientes somáticos. In OSÓRIO, L. C. Grupoterapia Hoje. Porto Alegre: Artes Médicas, 1986.

22. MINAYO, M. C. S. O desafio do conhecimento. Pesquisa qualitativa em Saúde. São Paulo: Hucitec - Abrasco, 1992.

23. MUNARI, D. B. Atividades grupais realizadas por enfermeiras na assistência à saúde em Ribeirão Preto: um estudo preliminar. In: $46^{\mathrm{a}}$ Reunião Anual da Sociedade Brasileira para o Progresso da Ciência, 1994, Vitória. Anais ... Vitória: 1994. p. 75. 
24. OSÓRIO, L. C. et. al . Grupoterapia Hoje. Porto Alegre: Artes Médicas, 1986.

25. PAYNE, J. A . The Contribuition do Group Learning to be Reahabilitation of Spinal Cord Injured Adults. Rehabilitation Nursing, v. 18, n. 6, nov./dec., 1993.

26. SANTOS, B. R. L. \& MENDES, E. E. M. Programa de assistência de enfermagem a clientes portadores de danos cardiovasculares, no ambulatório de um Hospital Geral e de Ensino de Porto Alegre - R. S. R. Bras. Enferm. Brasília, v. 36, n. 3/4, p. 274-81, 1983.

27. SCAIN, S. N. Educação para a saúde e grupos de clientes diabéticos. R. Gau. Enferm. Porto Alegre, v. 7, n. 2, p. 232-46, 1986.

28. SCHIMIDT, M. J. \& FREDDI, W. E. S. Preparo da gestante para o parto: aulas teórico-práticas. R. Bras. Enferm. Brasília, v. 28, n. 1, p. 15-25, 1975.

29. SELLtiz, C. e cols. Métodos de Pesquisa nas Relações Sociais. São Paulo: EPU / EDUSP, 1975.

30. TAFT, L. B. Computer-Assisted qualitative research. Research in Nursing \& Health, v. 16, p. 379-383, 1993.

31. VARGAS, G. O . P. \& SCAIN, S. F. Educação para a saúde a grupos de obesos. R. Bras. Enferm. Brasília, v. 37, n. 1, p. 38-49, 1983.

32. WILSON, M. - Group Theory / Process of nursing practice. USA: Bowie Brooly Communications Companu. Inc, 1985.

33. WOOD, J. K. Pequenos grupos centrados na pessoa: mais que terapia.campinas: PCSG, 1990. (mimeografado)

34. YALOM, I. D. The Theory and Practice of Group Psychotherapy. New York / London: Basic Books Inc. Publishers, 1975.

35. ZAGO, M. F. O . O Ritual de Orientação de pacientes pelos Enfermeiros Cirúrgicos: um estudo etnográfico. Ribeirão Preto, 1994. 154 p. Tese (Doutorado) - Escola de Enfermagem de Rịbeirão Preto, Universidade de São Paulo. 